\title{
Synthesis of Ferrocene/hydrofullerene Hybrid and Functionalized Bucky Ferrocenes
}

\author{
Motoki Toganoh, Yutaka Matsuo and Eiichi Nakamura* \\ Department of Chemistry, The University of Tokyo, Bunkyo-ku, Tokyo 113-0033, Japan \\ e-mail:nakamura@chem.s.u-tokyo.ac.jp
}

\section{Table of Contents}

1. Experimental Section $\quad \ldots \ldots . \quad$ S1

2. $\quad{ }^{1} \mathrm{H}$ NMR Spectra of $\mathbf{6}$ and Deprotonated Products $\quad \ldots . . \quad$ S11

\section{Experimental Section}

General. All reactions were carried out in an oven-dried reaction vessel under argon or nitrogen and were analyzed by HPLC (column: Buckyprep, 4.6 x $250 \mathrm{~mm}$, Nacalai tesque; flow rate: $1.0 \mathrm{~mL} / \mathrm{min}$; eluent: toluene/2-propanol = 7/3; detector: SPD-M10Avp, Shimadzu). The isolated yields were calculated on the basis of the starting fullerene compounds. All ${ }^{1} \mathrm{H}$ NMR spectra were measured at $400 \mathrm{MHz}$ (JEOL EX-400), and ${ }^{13} \mathrm{C}$ NMR spectra at $100 \mathrm{MHz}$. Spectra are reported in part per million from internal tetramethylsilane $(\delta 0.00 \mathrm{ppm})$ for ${ }^{1} \mathrm{H}$ NMR and from solvent carbon (e.g. $\delta 77.00 \mathrm{ppm}$ for chloroform) for ${ }^{13} \mathrm{C}$ NMR. IR spectra were recorded on Applied Systems Inc., REACT IR 1000 as powder on a diamond probe, or on a JASCO IR-420 instrument as a $\mathrm{KBr}$ disk; absorptions are reported in $\mathrm{cm}^{-1}$. Mass spectra were measured with Waters ZQ2000 (APCI mode) equipped with a Buckyprep column. High resolution mass spectra were recorded on JEOL JMS-T100LC instrument. Preparative HPLC was performed on a Buckyprep column (20 x $250 \mathrm{~mm}$ ) using toluene/2-propanol = 7/3, $6 / 4$ or $5 / 5$ as eluent (flow rate $20 \mathrm{~mL} / \mathrm{min}$, detected at $350 \mathrm{~nm}$ with an $\mathrm{UV}$ spectrophotometric detector, Shimadzu SPD-6A). 
Solvents and Materials. All commercially available reagents were distilled or recrystallized before use unless otherwise noted. $\mathrm{PhCN}$ was distilled under reduced pressure from $\mathrm{P}_{2} \mathrm{O}_{5}$ and stored under nitrogen. The use of molecular sieves should be avoided, since the reaction became irreproducible. The water content of the solvent was determined with a Karl-Fischer Moisture Titrator (MK-210, Kyoto Electronics Company) to be less than $30 \mathrm{ppm}$. $\left[\mathrm{Fe}\left(\mathrm{C}_{5} \mathrm{H}_{5}\right)(\mathrm{CO})_{2}\right]_{2}$ and TBAOH $(1 \mathrm{M}$ in $\mathrm{MeOH})$ were purchased from Acros Organics and used as received.

Synthesis of $\quad\left(\eta^{5}\right.$-cyclopentadienyl $)\left[(1,2,3,4,5-\eta)-9,12,15,18\right.$-tetrahydro $\left(\mathrm{C}_{60^{-}}\right.$ $\left.I_{\mathrm{h}}\right)[5,6]$ fulleren-1(6H)-yl]iron(II) $<\mathrm{Fe}\left(\mathrm{C}_{60} \mathrm{H}_{5}\right)\left(\mathrm{C}_{5} \mathrm{H}_{5}\right)(\mathbf{1})>$.

$\mathrm{C}_{60}\left(19.9 \mathrm{mg}, 27.6 \mu \mathrm{mol}, 1.00\right.$ equiv) and $\left[\mathrm{Fe}\left(\mathrm{C}_{5} \mathrm{H}_{5}\right)(\mathrm{CO})_{2}\right]_{2}(51.7 \mathrm{mg}, 146 \mu \mathrm{mol}$, 5.29 equiv) in $\mathrm{PhCN}(2.00 \mathrm{~mL})$ was degassed under reduced pressure $(\sim 0.3 \mathrm{mmHg})$ over 30 min at $0{ }^{\circ} \mathrm{C}$ and the resulting mixture was stirred at $160{ }^{\circ} \mathrm{C}$ for $22 \mathrm{~h}$. The reaction mixture was diluted with $8 \mathrm{~mL}$ of toluene/2-propanol $=7 / 3$, filtered with a pad of Celite and subjected to preparative HPLC separation. A collected fraction was concentrated to dryness and dried under reduced pressure to give $\mathrm{Fe}\left(\mathrm{C}_{60} \mathrm{H}_{5}\right)\left(\mathrm{C}_{5} \mathrm{H}_{5}\right)(\mathbf{1})$ as a brown solid $(5.9 \mathrm{mg}, 25 \%$ yield $) .{ }^{1} \mathrm{H}$ $\operatorname{NMR}\left(\mathrm{CS}_{2}, 400 \mathrm{MHz}\right) \delta 4.94\left(\mathrm{~s}, 5 \mathrm{H}, \mathrm{C}_{5} \mathrm{H}_{5}\right), 5.42\left(\mathrm{~s}, 5 \mathrm{H}, \mathrm{C}_{60} \mathrm{H}_{5}\right) ;{ }^{13} \mathrm{C} \mathrm{NMR}\left(\mathrm{CS}_{2}, 100 \mathrm{MHz}\right) \delta$ $45.39\left(\mathrm{~d},{ }^{1} J_{\mathrm{CH}}=143 \mathrm{~Hz}, 5 \mathrm{C}\right), 71.11(5 \mathrm{C}), 85.32(5 \mathrm{C}), 143.91$ (10C), 145.68 (10C), 147.13 (5C), 148.48 (10C), 148.50 (5C), 151.85 (10C); HRMS (APCI+) calcd for $\mathrm{C}_{65} \mathrm{H}_{11}{ }^{56} \mathrm{Fe}$ $\left(\mathrm{M}+\mathrm{H}^{+}\right)$: 847.0210, found: 847.0206.

Synthesis of $\left[(1,2,3,4,5-\eta)-6,9-d i b e n z y l-9,12,15,18\right.$-tetrahydro $\left(C_{60}-I_{h}\right)[5,6]$ fulleren- $1(6 H)$ yl $]\left(\eta^{5}\right.$-cyclopentadienyl)iron(II) $<\mathrm{Fe}\left[\mathrm{C}_{60}\left(\mathrm{PhCH}_{2}\right)_{2} \mathrm{H}_{3}\right]\left(\mathrm{C}_{5} \mathrm{H}_{5}\right)(5)>$.

$\mathrm{C}_{60}\left(\mathrm{PhCH}_{2}\right)_{2}\left(3,100 \mathrm{mg}, 111 \mu \mathrm{mol}, 1.00\right.$ equiv) and $\left[\mathrm{Fe}\left(\mathrm{C}_{5} \mathrm{H}_{5}\right)(\mathrm{CO})_{2}\right]_{2}(378 \mathrm{mg}, 1.07$ mmol, 9.64 equiv) in $\mathrm{PhCN}(20.0 \mathrm{~mL})$ was degassed under reduced pressure $(\sim 0.3 \mathrm{mmHg})$ at $0{ }^{\circ} \mathrm{C}$ for $40 \mathrm{~min}$ and the resulting mixture was heated at $160{ }^{\circ} \mathrm{C}$ for $90 \mathrm{~h}$. The reaction mixture was concentrated to dryness under reduced pressure and the residue was subjected to preparative HPLC separation. Collected fractions were concentrated to dryness and dried under reduced pressure to give 5 as an orange solid (50.3 mg, $44 \%$ yield). ${ }^{1} \mathrm{H} \mathrm{NMR}\left(\mathrm{CDCl}_{3}\right.$, $400 \mathrm{MHz}) \delta 4.34$ (d, $J=13.0 \mathrm{~Hz}, 2 \mathrm{H}, \mathrm{PhCH} H), 4.39$ (d, $J=13.0 \mathrm{~Hz}, 2 \mathrm{H}, \mathrm{PhCH} H), 5.11$ (s, $\left.5 \mathrm{H}, \mathrm{C}_{5} \mathrm{H}_{5}\right), 5.48\left(\mathrm{~d}, J=2.4 \mathrm{~Hz}, 1 \mathrm{H}, \mathrm{C}_{60} \mathrm{H}\right), 5.52\left(\mathrm{~d}, J=2.4 \mathrm{~Hz}, 2 \mathrm{H}, \mathrm{C}_{60} \mathrm{H}\right), 7.46-7.62(\mathrm{~m}, 10 \mathrm{H}$, $\mathrm{Ph}) ;{ }^{13} \mathrm{C}$ NMR $\left(\mathrm{CS}_{2}, 100 \mathrm{MHz}\right) \delta 45.08$ (2C), 45.39 (1C), 51.03 (2C), 56.02 (2C), 70.56

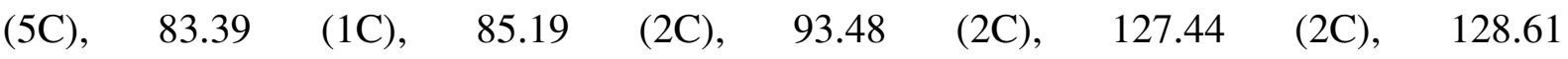
(4C), 130.62 (4C), 136.92 (2C), 142.81 (2C), 143.08 (2C), 143.63 (2C), 143.82 (2C), 143.97 (2C), 144.70 (2C), 145.08 (2C), 145.59 (2C), 145.71 (2C), 145.72 (2C), 147.00 (2C), 147.04 (2C), 147.08 (1C), 148.11 (2C), 148.19 (2C), 148.23 (2C), 148.27 (2C), 148.29 (2C), 148.40 (2C+1C), 148.46 (2C), 150.64 (2C), 151.38 (2C), 151.79 (2C), 153.35 (2C), 153.37 (2C); HRMS (APCI+) calcd for $\mathrm{C}_{79} \mathrm{H}_{22}{ }^{56} \mathrm{Fe}\left(\mathrm{M}^{+}\right)$: 1026.1071, found: 1026.1110 . 
Synthesis of $\quad\left[(1,2,3,4,5-\eta)-6,9-d i b e n z y l-12-p h e n y l-9,12,15,18\right.$-tetrahydro $\left(C_{60^{-}}\right.$ $\left.I_{\mathrm{h}}\right)[5,6]$ fulleren-1 $(6 H)$-yl $]\left(\eta^{5}\right.$-cyclopentadienyl)iron(II) $<\mathrm{Fe}\left[\mathrm{C}_{60}\left(\mathrm{PhCH}_{2}\right)_{2} \mathrm{PhH}_{2}\right]\left(\mathrm{C}_{5} \mathrm{H}_{5}\right)(6)>$. $\mathrm{C}_{60}\left(\mathrm{PhCH}_{2}\right)_{2} \mathrm{PhH}\left(4,101 \mathrm{mg}, 103 \mu \mathrm{mol}, 1.00\right.$ equiv) and $\left[\mathrm{Fe}\left(\mathrm{C}_{5} \mathrm{H}_{5}\right)(\mathrm{CO})_{2}\right]_{2}(37.0$ $\mathrm{mg}, 104 \mu \mathrm{mol}, 1.01$ equiv) in $\mathrm{PhCN}(20.0 \mathrm{~mL})$ was degassed under reduced pressure $(\sim 0.3$ $\mathrm{mmHg}$ ) at $0{ }^{\circ} \mathrm{C}$ for $30 \mathrm{~min}$ and the resulting mixture was stirred at $160^{\circ} \mathrm{C}$ for $14 \mathrm{~h}$. The reaction mixture was concentrated to dryness under reduced pressure. Purification of the product was performed by preparative HPLC. Collected fractions were concentrated to a small volume and precipitated with methanol. Precipitates were collected by filtration, washed with methanol and dried under reduced pressure to give 5 as an orange solid (93.5 mg, 82\% yield). IR (KBr disk) 3085 (w), 3060 (w), 3027 (w), 2959 (w), 2918 (m), 2850 (w), 1494 (s), 1454 (s), 1418 (m), 1384 (m), 1286 (w), 1261 (s), 1236 (w), 1198 (w), 1177 (w), 1156 (w), 1106 (s), $1030(\mathrm{~m}), 908$ (w), 822 (m), 804 (m), 767 (w), $744(\mathrm{w}), 723$ (w), 698 (s), $674(\mathrm{w}), 551$ (m), $508(\mathrm{~m}) ;{ }^{1} \mathrm{H}$ NMR $\left(\mathrm{CDCl}_{3}, 400 \mathrm{MHz}\right) \delta 4.06$ (s, 2H, PhCH $\left.\mathrm{H}_{2}\right), 4.07$ (d, $J$ $=13.2 \mathrm{~Hz}, 1 \mathrm{H}, \mathrm{PhCH} H), 4.20(\mathrm{~d}, J=13.2 \mathrm{~Hz}, 1 \mathrm{H}, \mathrm{PhCH} H), 4.33\left(\mathrm{~s}, 5 \mathrm{H}, \mathrm{C}_{5} \mathrm{H}_{5}\right), 5.26(\mathrm{~d}, J=$ $\left.2.8 \mathrm{~Hz}, 1 \mathrm{H}, \mathrm{C}_{60} \mathrm{H}\right), 5.42\left(\mathrm{~d}, J=2.8 \mathrm{~Hz}, 1 \mathrm{H}, \mathrm{C}_{60} \mathrm{H}\right), 7.26-7.39(\mathrm{~m}, 8 \mathrm{H}, \mathrm{Ph}), 7.48-7.54(\mathrm{~m}, 3 \mathrm{H}$, $\mathrm{Ph}$ ), $7.70(\mathrm{t}, J=7.8 \mathrm{HZ}, 2 \mathrm{H}, \mathrm{Ph}), 8.16(\mathrm{dd}, J=0.8,8.0 \mathrm{~Hz}, 2 \mathrm{H}, \mathrm{Ph}) ;{ }^{13} \mathrm{C} \mathrm{NMR}\left(\mathrm{CS}_{2}, 100\right.$ $\mathrm{MHz}) \delta 45.09\left(\mathrm{~d},{ }^{1} J_{\mathrm{CH}}=142 \mathrm{~Hz}, 1 \mathrm{C}\right), 45.39\left(\mathrm{~d},{ }^{1} J_{\mathrm{CH}}=142 \mathrm{~Hz}, 1 \mathrm{C}\right), 50.16\left(\mathrm{t},{ }^{1} J_{\mathrm{CH}}=131\right.$ $\mathrm{Hz}, 1 \mathrm{C}), 51.09\left(\mathrm{t},{ }^{1} J_{\mathrm{CH}}=131 \mathrm{~Hz}, 1 \mathrm{C}\right), 55.98$ (1C), 56.02 (1C), 58.58 (1C), 70.57 (5C), 83.67 (1C), 84.03 (1C), 92.11 (1C), 93.75 (1C), 94.33 (1C), 127.40 (1C), 127.51 (1C), 127.80 (1C), 127.90 (2C), 128.57 (2C), 128.68 (2C), 129.21 (2C), 130.56 (2C), 130.59 (2C), 136.81 (1C), 136.97 (1C), 142.76 (1C), 142.84 (1C), 142.94 (1C), 143.08 (1C), 143.34 (1C), 143.55 (1C), 143.65 (1C), 143.66 (1C), 143.70 (1C), 143.88 (1C), 143.89 (1C), 144.18 (1C), 144.59 (1C), 144.69 (1C), 144.90 (1C), 144.94 (1C), 145.06 (1C), 145.58 (1C+1C), 145.80 (1C), 145.82 (1C), 146.97 (1C), 147.05 (1C+1C), 147.13 (1C), 147.26 (1C), 148.08 (1C), $148.11(1 \mathrm{C}+1 \mathrm{C}), 148.12(1 \mathrm{C}), 148.16(1 \mathrm{C}), 148.22(1 \mathrm{C}+1 \mathrm{C}+1 \mathrm{C}), 148.23$ (1C), 148.28 (1C), 148.39 (1C), 148.42 (1C), 148.45 (1C), 148.46 (1C), 148.50 (1C), 150.62 (1C), 150.84 (1C), 151.27 (1C), 151.56 (1C), 151.93 (1C), 152.95 (1C), 153.11 (1C), 153.43 (1C), 153.83 (1C), 153.87 (1C); Anal. Calcd for 6: C, 92.56; H, 2.38. Found: C, 92.44; H. 2.63; HRMS (APCI+) calcd for $\mathrm{C}_{85} \mathrm{H}_{26}{ }^{56} \mathrm{Fe}\left(\mathrm{M}^{+}\right)$: 1102.1384, found: 1102.1396 .

Synthesis of $\quad\left[(1,2,3,4,5-\eta)\right.$-6,9-dibenzyl-12-phenyl-(9,12,15,18-tetrahydro $\left(\mathrm{C}_{60^{-}}\right.$ $\left.I_{\mathrm{h}}\right)[5,6]$ fulleren-1 $\left.(6 H)-y \mathrm{l}\right]\left[\eta^{5}\right.$-(1,3-bis(trimethlysilyl))cyclopentadienyl]iron(II) $<\mathrm{Fe}\left[\mathrm{C}_{60}\left(\mathrm{PhCH}_{2}\right)_{2} \mathrm{PhH}_{2}\right]\left[\left(1,3-\mathrm{Me}_{3} \mathrm{Si}\right)_{2} \mathrm{C}_{5} \mathrm{H}_{3}\right](7)>$.

A mixture of $\mathrm{C}_{60}\left(\mathrm{PhCH}_{2}\right)_{2} \mathrm{PhH}\left(\mathbf{4}, 19.7 \mathrm{mg}, 20.1 \mathrm{mmol}, 1.00\right.$ equiv) and $\left[\mathrm{Fe}\left(\mathrm{C}_{5}(1,3-\right.\right.$ $\left.\left.\mathrm{Me}_{3} \mathrm{Si}_{2} \mathrm{H}_{3}\right)(\mathrm{CO})_{2}\right]_{2}(13.2 \mathrm{mg}, 20.5 \mathrm{mmol}, 1.02$ equiv) in $\mathrm{PhCN}$ (4.0 mL) was degassed under reduced pressure $(\sim 0.3 \mathrm{mmHg})$ at $0{ }^{\circ} \mathrm{C}$ for $30 \mathrm{~min}$ and the resulting mixture was stirred at $160{ }^{\circ} \mathrm{C}$ for $24 \mathrm{~h}$. The resulting reaction mixture was diluted with toluene/2-propanol=1/1 
and filtered through a pad of Celite. The filtrate was subjected into preparative HPLC separation. The collected fractions were concentrated to dryness and dried under reduced pressure to give $\mathrm{Fe}\left[\mathrm{C}_{60}\left(\mathrm{PhCH}_{2}\right)_{2} \mathrm{PhH}_{2}\right]\left[\left(1,3-\mathrm{Me}_{3} \mathrm{Si}\right)_{2} \mathrm{C}_{5} \mathrm{H}_{3}\right](7)$ as a red solid $(13.2 \mathrm{mg}, 53 \%$ yield). ${ }^{1} \mathrm{H} \mathrm{NMR}\left(\mathrm{CS}_{2}, 400 \mathrm{MHz}\right) \delta 0.58$ (s, 9H, $\mathrm{Me}_{3} \mathrm{Si}$ ), 0.83 (s, 9H, $\mathrm{Me}_{3} \mathrm{Si}$ ), 3.53 (s, 1H, CpH), $4.20(\mathrm{~d}, J=13.2 \mathrm{~Hz}, 1 \mathrm{H}, \mathrm{PhCH} H), 4.36(\mathrm{~d}, J=13.2 \mathrm{~Hz}, 1 \mathrm{H}, \mathrm{PhCH} H), 4.40(\mathrm{~d}, J$ $=13.2 \mathrm{~Hz}, 1 \mathrm{H}, \mathrm{PhCH} H), 4.47(\mathrm{~d}, J=13.2 \mathrm{~Hz}, 1 \mathrm{H}, \mathrm{PhCH} H), 4.63(\mathrm{~s}, 1 \mathrm{H}+1 \mathrm{H}, \mathrm{Cp} H), 5.52(\mathrm{~d}, J$ $\left.=2.6 \mathrm{~Hz}, 1 \mathrm{H}, \mathrm{C}_{60} \mathrm{H}\right), 5.65\left(\mathrm{~d}, J=2.6 \mathrm{~Hz}, 1 \mathrm{H}, \mathrm{C}_{60} \mathrm{H}\right), 7.44-7.81(\mathrm{~m}, 10 \mathrm{H}, \mathrm{Ph}), 7.81(\mathrm{t}, J=7.5$ $\mathrm{Hz}, 1 \mathrm{H}, \mathrm{Ph}), 7.96(\mathrm{t}, J=7.5 \mathrm{~Hz}, 2 \mathrm{H}, \mathrm{Ph}), 8.40(\mathrm{~d}, J=7.5 \mathrm{~Hz}, 2 \mathrm{H}, \mathrm{Ph}) ;{ }^{13} \mathrm{C} \mathrm{NMR}\left(\mathrm{CS}_{2}, 100\right.$ $\mathrm{MHz}) \delta 0.87$ (3C), 1.38 (3C), 45.39 (1C), 45.76 (1C), 51.63 (1C), 51.70 (1C), 55.34 (1C), 55.70 (1C), 58.62 (1C), 77.54 (1C), 77.60 (1C), 77.97 (1C), 78.67 (1C), 80.66 (1C), 82.43 (1C), 83.08 (1C), 93.11 (1C), 94.02 (1C), 5.77 (1C), 127.43 (1C), 127.63 (1C), 127.83 (1C), 128.14 (2C), 128.64 (2C), 128.80 (2C), 129.27 (2C), 130.55 (2C), 130.65 (2C), 136.68 (1C), 136.80 (1C), 142.87 (1C), 142.93 (1C), 143.07 (1C), 143.08 (1C), 143.38 (1C), 143.56 (1C), 143.61 (1C), 143.72 (1C), 143.83 (1C), 143.96 (1C), 144.02 (1C), 144.20 (1C), 144.58 (1C), 144.66 (1C), 144.78 (1C), 145.04 (1C), 145.08 (1C), 145.46 (1C), 145.72 (1C), 145.73 (1C), 147.02 (1C), 147.07 (1C), 147.11 (1C), 147.18 (1C), 147.22 (1C), 147.28 (1C), 148.16 (1C), 148.18 (1C), 148.21 (1C), 148.22 (1C), 148.24 (1C), 148.28 (1C), 148.30 (1C), 148.32 (1C), 148.37 (1C), 148.43 (1C), 148.45 (1C), 148.47 (1C), 148.52 (1C), 158.56 (1C), 148.58 (1C), 150.10 (1C), 150.48 (1C), 150.97 (1C), 151.21 (1C), 151.43 (1C), 152.59 (1C), 152.79 (1C), 153.33 (1C), 153.62 (1C), 153.88 (1C); HRMS (APCI+) calcd for $\mathrm{C}_{91} \mathrm{H}_{42}{ }^{56} \mathrm{Fe}^{28} \mathrm{Si}_{2}\left(\mathrm{M}^{+}\right): 1246.2174$, found: 1246.2141 .

\section{Deprotonation of 6.}

To a solution of $6(2.1 \mathrm{mg}, 1.9 \mu \mathrm{mol})$ in $\mathrm{PhCN}-d_{5}(0.60 \mathrm{~mL})$ in an NMR tube was added a solution of tetrabutylammonium hydroxide in $\mathrm{MeOH}(1.0 \mathrm{M}, 6.0 \mu \mathrm{l}, 3.2$ equiv) at $21{ }^{\circ} \mathrm{C}$ under nitrogen. The color of the solution changed from orange to dark brown within a few seconds. This sample was directly used for NMR analysis. ${ }^{1} \mathrm{H}$ NMR $\left(\mathrm{PhCN}-d_{5}, 400\right.$ $\mathrm{MHz}) \delta 4.40(\mathrm{~d}, J=13.0 \mathrm{~Hz}, 1 \mathrm{H}, \mathrm{PhCH} H), 4.53(\mathrm{~d}, J=13.0 \mathrm{~Hz}, 1 \mathrm{H}, \mathrm{PhCH} H), 4.74$ (d, $J$ $=13.0 \mathrm{~Hz}, 1 \mathrm{H}, \mathrm{PhCH} H), 4.75(\mathrm{~d}, J=13.0 \mathrm{~Hz}, 1 \mathrm{H}, \mathrm{PhCH} H), 5.26(\mathrm{~s}, 5 \mathrm{H}, \mathrm{Cp}), 7.91(\mathrm{~d}, J=7.4$ $\mathrm{Hz}, 2 \mathrm{H}, \mathrm{Ph}), 8.09$ (d, $J=7.8 \mathrm{~Hz}, 2 \mathrm{H}, \mathrm{Ph}), 8.72(\mathrm{~d}, J=7.8 \mathrm{~Hz}, 2 \mathrm{H}, \mathrm{Ph}$ ) [Signals of the other aromatic protons overlapped with those of the solvent.].

cf. The ${ }^{1} \mathrm{H}$ NMR spectrum of 4 in $\mathrm{PhCN}-d_{5}:{ }^{1} \mathrm{H}$ NMR $\left(\mathrm{PhCN}-d_{5}, 400 \mathrm{MHz}\right) \delta 4.29$ (d, $J=13.2 \mathrm{~Hz}, 1 \mathrm{H}, \mathrm{PhCH} H), 4.33(\mathrm{~d}, J=13.4 \mathrm{~Hz}, 1 \mathrm{H}, \mathrm{PhCH} H), 4.37(\mathrm{~d}, J=13.4 \mathrm{~Hz}, 1 \mathrm{H}$, $\mathrm{PhCH} H), 4.39$ (d, $J=13.2 \mathrm{~Hz}, 1 \mathrm{H}, \mathrm{PhCH} H), 4.58$ (s, 5H, Cp), $5.48\left(\mathrm{~d}, J=2.6 \mathrm{~Hz}, 1 \mathrm{H}, \mathrm{C}_{60} \mathrm{H}\right)$, $5.55\left(\mathrm{~d}, J=2.6 \mathrm{~Hz}, 1 \mathrm{H}, \mathrm{C}_{60} \mathrm{H}\right), 7.79(\mathrm{~d}, J=7.2 \mathrm{~Hz}, 2 \mathrm{H}, \mathrm{Ph}), 7.88(\mathrm{t}, J=7.8 \mathrm{~Hz}, 2 \mathrm{H}$, $\mathrm{Ph}), 8.32(\mathrm{dd}, J=0.8,8.2 \mathrm{~Hz}, 2 \mathrm{H}, \mathrm{Ph})$ [Signals of the other aromatic protons overlapped with those of the solvent.]. 


\section{Typical procedures of alkylation of 6.}

A solution of tetrabutylammonium hydroxide (1.0 $\mathrm{M}$ in $\mathrm{MeOH}, 3.1$ equiv) was added to a solution of $\mathrm{Fe}\left[\mathrm{C}_{60}\left(\mathrm{PhCH}_{2}\right)_{2} \mathrm{PhH}_{2}\right]\left(\mathrm{C}_{5} \mathrm{H}_{5}\right)\left(6,1.0\right.$ equiv) at room temperature $\left(21 \sim 26^{\circ} \mathrm{C}\right)$. $\mathrm{A}$ color of the resulting solution changed from orange to dark red at a moment. After $5 \mathrm{~min}$, the mixture was treated with alkyl halide (RX, 5 to 40 equiv, depending on RX), resulting change of color from dark red to brown. HPLC analysis indicated the full consumption of the starting material within 10 minutes. Then the mixture was diluted with $8 \mathrm{~mL}$ of toluene/2-propanol $=1 / 1$, filtered with a pad of alumina and subjected to preparative HPLC separation. A collected fraction was concentrated to dryness and dried under reduced pressure to give a dialkylated product $\mathrm{Fe}\left[\left(\mathrm{C}_{60}\left(\mathrm{PhCH}_{2}\right)_{2} \mathrm{PhR}_{2}\right]\left(\mathrm{C}_{5} \mathrm{H}_{5}\right)\right.$.

Synthesis of $\quad[(1,2,3,4,5-\eta)-6,9-d i b e n z y-12,15$-dimethyl-18-phenyl-9,12,15,18tetrahydro $\left(\mathrm{C}_{60}-I_{\mathrm{h}}\right)[5,6]$ fulleren-1 $\left.(6 H)-\mathrm{yl}\right]\left(\eta^{5}\right.$-cyclopentadienyl)iron(II)

$<\mathrm{Fe}\left[\mathrm{C}_{60}\left(\mathrm{PhCH}_{2}\right)_{2} \mathrm{Me}_{2} \mathrm{Ph}\right]\left(\mathrm{C}_{5} \mathrm{H}_{5}\right)(8)>$.

The procedures $(\mathrm{RX}=\mathrm{MeI})$ described above was performed to obtain 8 . Yield: 81\%; ${ }^{1} \mathrm{H}$ NMR $\left(\mathrm{CS}_{2}, 400 \mathrm{MHz}\right) \delta 1.86$ (s, 3H, Me), 1.97 (s, 3H, Me), 3.26 (d, $J=12.8$ $\mathrm{Hz}, 1 \mathrm{H}, \mathrm{PhCH} H$ ), 3.32 (d, $J=13.2 \mathrm{~Hz}, 1 \mathrm{H}, \mathrm{PhCH} H$ ), 3.33 (d, $J=12.8 \mathrm{~Hz}, 1 \mathrm{H}, \mathrm{PhCH} H$ ), $3.59(1 \mathrm{H}, \mathrm{PhCH} H)$ [This signal overlaps with the signal due to the $\mathrm{Cp}$ moiety.], $3.62(\mathrm{~s}, 5 \mathrm{H}$, Cp), 6.44-6.59 (m, 8H, Ph), $6.70(\mathrm{~d}, J=7.0 \mathrm{~Hz}, 2 \mathrm{H}, \mathrm{Ph}), 6.78$ (t, $J=7.4 \mathrm{~Hz}, 1 \mathrm{H}, \mathrm{Ph}), 6.96$ (t, $J=7.6 \mathrm{~Hz}, 2 \mathrm{H}, \mathrm{Ph}), 7.46(\mathrm{~d}, J=7.3 \mathrm{~Hz}, 2 \mathrm{H}, \mathrm{Ph}) ;{ }^{13} \mathrm{C} \mathrm{NMR}\left(\mathrm{CS}_{2}, 100 \mathrm{MHz}\right) \delta 29.27(1 \mathrm{C})$, 29.82 (1C), 49.93 (1C), 50.38 (1C), 50.44 (1C), 51.08 (1C), 55.65 (1C), 55.78 (1C), 58.58 (1C), 69.50 (5C), 91.15 (1C), 91.21 (1C), 92.11 (1C), 92.17 (1C), 93.22 (1C), 127.18 (1C), 127.32 (1C), 127.59 (1C), 128.23 (2C), 128.35 (2C), 128.48 (2C), 128.94 (2C), 130.36 (2C), 130.40 (2C), 136.51 (1C), 136.60 (1C), 142.55 (1C), 142.56 (1C), 142.62 (1C), 142.64 (1C), 143.09 (1C), 143.10 (1C), 143.26 (1C), 143.31 (1C), 143.37 (1C), 143.38 (1C), 143.60 (1C), $143.76(1 \mathrm{C}+1 \mathrm{C}), 143.81(1 \mathrm{C}+1 \mathrm{C}), 144.07$ (1C), 144.16 (1C), 144.43 (1C), 144.75 (1C), 144.83 (1C), 144.99 (1C), 146.75 (1C), 146.837 (1C), 146.842 (1C), 146.98

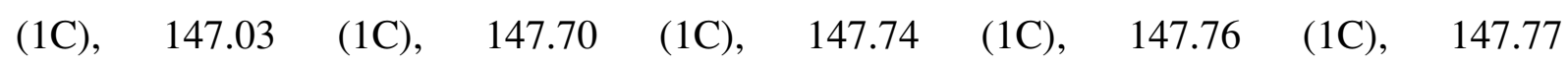
(1C), 147.79 (1C), 147.82 (1C), 147.90 (1C+1C), 147.95 (1C), 148.00 (1C), 148.10 (1C), 148.16 (1C), 148.24 (1C), 148.25 (1C), 148.29 (1C), 151.88 (1C), 152.04 (1C), 152.11 (1C), 152.35 (1C), 152.41 (1C), 152.94 (1C), 153.87 (1C), 154.63 (1C), 154.80 (1C), 155.02 (1C); HRMS (APCI+) calcd for $\mathrm{C}_{87} \mathrm{H}_{30}{ }^{56} \mathrm{Fe}\left(\mathrm{M}^{+}\right)$: 1130.1697 , found: 1130.1708 .

Synthesis of $\quad[(1,2,3,4,5-\eta)-6,9$-diallyl-12,15-dibenzyl-18-phenyl-9,12,15,18tetrahydro $\left(\mathrm{C}_{60}-I_{\mathrm{h}}\right)[5,6]$ fulleren-1 $\left.(6 H)-\mathrm{yl}\right]\left(\eta^{5}\right.$-cyclopentadienyl)iron(II) $<\mathrm{Fe}\left[\mathrm{C}_{60}(\text { allyl })_{2}\left(\mathrm{PhCH}_{2}\right)_{2} \mathrm{Ph}\right]\left(\mathrm{C}_{5} \mathrm{H}_{5}\right)(9)>$.

The procedures $(\mathrm{RX}=$ allyl bromide) described above was performed to obtain 9. Yield: 77\%; ${ }^{1} \mathrm{H}$ NMR $\left(\mathrm{CS}_{2}, 400 \mathrm{MHz}\right) \delta 2.80\left(\mathrm{~d}, J=7.0 \mathrm{~Hz}, 2 \mathrm{H}, \mathrm{H}_{2} \mathrm{C}=\mathrm{CH}-\mathrm{CH}_{2}\right), 2.81$ (dd, $J=$ 
$\left.6.5,13.8 \mathrm{~Hz}, 1 \mathrm{H}, \mathrm{H}_{2} \mathrm{C}=\mathrm{CH}-\mathrm{CH} H\right), 3.03\left(\mathrm{dd}, J=7.7,13.8 \mathrm{~Hz}, 1 \mathrm{H}, \mathrm{H}_{2} \mathrm{C}=\mathrm{CH}-\mathrm{CH} H\right), 3.27(\mathrm{~d}, J$ $=13.0 \mathrm{~Hz}, 1 \mathrm{H}, \mathrm{PhCH} H), 3.32(\mathrm{~d}, J=13.0 \mathrm{~Hz}, 1 \mathrm{H}, \mathrm{PhCH} H), 3.33(\mathrm{~d}, J=13.2 \mathrm{~Hz}, 1 \mathrm{H}$, $\mathrm{PhCH} H), 3.602$ (s, 2H, CpH), $3.603(\mathrm{~s}, 2 \mathrm{H}, \mathrm{CpH}), 3.604$ (s, 1H, CpH), 3.61 (d, J $=13.2 \mathrm{~Hz}, 1 \mathrm{H}, \mathrm{PhCH} H), 4.62\left(\mathrm{~d}, J=10.0 \mathrm{~Hz}, 2 \mathrm{H}, \mathrm{H} H \mathrm{C}=\mathrm{CH}-\mathrm{CH}_{2}\right), 4.68(\mathrm{~d}, J=17.0 \mathrm{~Hz}, 1 \mathrm{H}$, $\left.\mathrm{H} H \mathrm{C}=\mathrm{CH}-\mathrm{CH}_{2}\right), 4.72\left(\mathrm{~d}, J=16.8 \mathrm{~Hz}, 1 \mathrm{H}, \mathrm{H} H \mathrm{C}=\mathrm{CH}-\mathrm{CH}_{2}\right), 5.62-5.74\left(\mathrm{~m}, 2 \mathrm{H}, \mathrm{H}_{2} \mathrm{C}=\mathrm{CH}-\mathrm{CH}_{2}\right)$, 6.42-6.58 (m, 8H, Ph), 6.69 (d, $J=7.0 \mathrm{~Hz}, 2 \mathrm{H}, \mathrm{Ph}), 6.78$ (t, $J=7.4 \mathrm{~Hz}, 1 \mathrm{H}, \mathrm{Ph}), 6.97$ (t, $J=$ $7.8 \mathrm{~Hz}, 2 \mathrm{H}, \mathrm{Ph}), 7.44$ (d, $J=7.8 \mathrm{~Hz}, 2 \mathrm{H}, \mathrm{Ph}) ;{ }^{13} \mathrm{C} \mathrm{NMR}\left(\mathrm{CS}_{2}, 100 \mathrm{MHz}\right) \delta 47.36(1 \mathrm{C}), 48.04$ (1C), 49.96 (1C), 50.98 (1C), 54.48 (1C), 54.60 (1C), 55.65 (1C), 55.72 (1C), 58.51 (1C), 69.78 (5C), 90.88 (1C), 91.28 (1C), 91.38 (1C), 92.37 (1C), 93.20 (1C), 119.32 (1C), 119.37 (1C), 127.21 (1C), 127.34 (1C), 127.65 (1C), 128.20 (2C), 128.33 (2C), 128.47 (2C), 128.94 (2C), 130.37 (2C), 130.40 (2C), 134.24 (1C), 134.52 (1C), 136.45 (1C), 136.53 (1C), 142.54 (1C), 142.58 (1C), 142.63 (1C), 142.65 (1C), 142.92 (1C+1C), $143.00(1 \mathrm{C}+1 \mathrm{C}), 143.11$

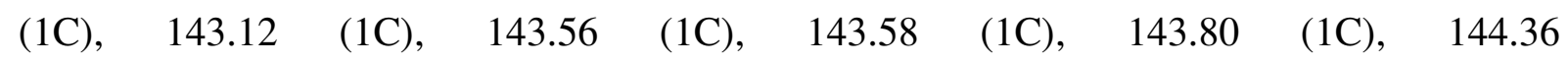
$(1 \mathrm{C}+1 \mathrm{C}), 144.49$ (1C), 144.58 (1C), 144.68 (1C), 144.71 (1C), 144.73 (1C), 144.97 (1C), 146.75 (1C), 146.85 (1C), 146. 90 (1C), 146.98 (1C), 147.03 (1C), 147.70 (1C), 147.75

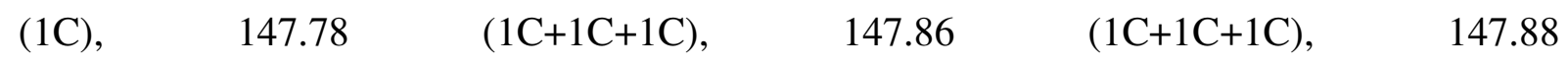
(1C), 147.92 (1C), 148.12 (1C), 148.17 (1C), 148.25 (1C+1C), 148.30 (1C), 151.85 (1C), 152.06 (1C), 152.22 (1C), 152.24 (1C), 152.30 (1C), 152.55 (1C), 152.70 $(1 \mathrm{C}+1 \mathrm{C}), 152.87(1 \mathrm{C}), 153.08(1 \mathrm{C})$; HRMS (APCI+) calcd for $\mathrm{C}_{91} \mathrm{H}_{35}{ }^{56} \mathrm{Fe}\left(\mathrm{M}+\mathrm{H}^{+}\right)$: 1183.2088, found: 1183.2046 .

Synthesis of $\left[(1,2,3,4,5-\eta)-6,9,12,15\right.$-tetrabenzyl-18-phenyl-9,12,15,18-tetrahydro $\left(C_{60^{-}}\right.$ $\left.I_{\mathrm{h}}\right)[5,6]$ fulleren-1 $(6 H)$-yl $]\left(\eta^{5}\right.$-cyclopentadienyl)iron(II) $<\mathrm{Fe}\left[\mathrm{C}_{60}\left(\mathrm{PhCH}_{2}\right)_{4} \mathrm{Ph}\right]\left(\mathrm{C}_{5} \mathrm{H}_{5}\right)(10)>$.

The procedures $\left(\mathrm{RX}=\mathrm{PhCH}_{2} \mathrm{Br}\right)$ described above was performed to obtain 10. Yield; 60\%; ${ }^{1} \mathrm{H} \mathrm{NMR}\left(\mathrm{CDCl}_{3}, 400 \mathrm{MHz}\right) \delta 4.18(\mathrm{~d}, J=13.2 \mathrm{~Hz}, 2 \mathrm{H}, \mathrm{PhCH} H), 4.20(\mathrm{~d}, J=13.2 \mathrm{~Hz}$, $2 \mathrm{H}, \mathrm{PhCH} H), 4.24(\mathrm{~d}, J=13.2 \mathrm{~Hz}, 2 \mathrm{H}, \mathrm{PhCH} H), 4.49$ (d, $J=13.2 \mathrm{~Hz}, 2 \mathrm{H}, \mathrm{PhCH} H), 4.54$ (s, $5 \mathrm{H}, \mathrm{C}_{5} \mathrm{H}_{5}$ ), 7.28-7.47 (m, 16H, Ph), 7.52-7.62 (m, 5H, Ph), $7.80(\mathrm{t}, J=7.8 \mathrm{~Hz}, 2 \mathrm{H}, \mathrm{Ph}), 8.43$ $(\mathrm{d}, J=7.2 \mathrm{~Hz}, 2 \mathrm{H}, \mathrm{Ph}) ;{ }^{13} \mathrm{C} \mathrm{NMR}\left(\mathrm{CDCl}_{3}, 100 \mathrm{MHz}\right) \delta 49.61(2 \mathrm{C}), 50.82(2 \mathrm{C}), 55.54(2 \mathrm{C})$, 55.61 (2C), 58.59 (1C), 69.42 (5C), 91.25 (2C), 92.21 (2C), 93.05 (1C), 126.97 (2C), 127.05 (2C), 127.49, 128.04 (2C), 128.15 (4C), 128.21 (4C), 128.81 (2C), 130.13 (4C), 130.17 (4C), 136.51 (2C), 136.58 (2C), 142.19 (2C), 142.22 (2C), 142.24 (2C), 142.25 (2C), 142.69 (2C), 143.37 (2C), 143.87 (1C), 144.19 (2C), 144.40 (2C), 144.52 (2C), 144.83 (2C), 146.56 (2C), 146.58 (1C), 146.75 (2C), 147.425 (2C), 147.434 (2C), 147.44

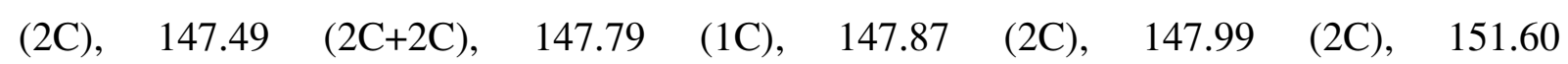
(2C), 152.02 (2C), 152.24 (2C), 152.38 (2C), 152.80 (2C); HRMS (APCI+) calcd for $\mathrm{C}_{99} \mathrm{H}_{38}{ }^{56} \mathrm{Fe}\left(\mathrm{M}^{+}\right):$1282.2323, found: 1282.2280 .

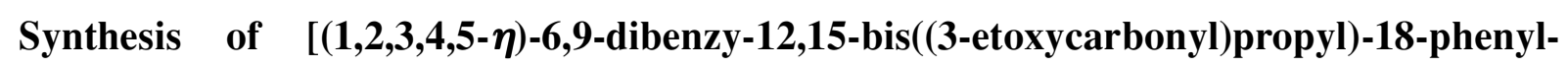




\section{9,12,15,18-tetrahydro $\left(\mathrm{C}_{60}-I_{\mathrm{h}}\right)[5,6]$ fulleren-1 $(6 H)$-yl $]\left(\eta^{5}\right.$-cyclopentadienyl)iron(II) $<\mathrm{Fe}\left[\mathrm{C}_{60}\left(\mathrm{PhCH}_{2}\right)_{2}\left(\mathrm{EtOOCCH}_{2} \mathrm{CH}_{2} \mathrm{CH}_{2}\right)_{2} \mathrm{Ph}\right]\left(\mathrm{C}_{5} \mathrm{H}_{5}\right)(11)>$.}

The procedures $\left(\mathrm{RX}=\mathrm{EtOOCCH}_{2} \mathrm{CH}_{2} \mathrm{CH}_{2} \mathrm{Br}\right)$ described above was performed to obtain 11. Yield: $64 \%$. When the same reaction using 6 (1.0 mg, $0.91 \mu \mathrm{mol})$, TBAOH (3.1 eq, $3.1 \mu \mathrm{L}, 1 \mathrm{M}$ solution of $\mathrm{MeOH}$ ), and $\mathrm{EtOOCCH}_{2} \mathrm{CH}_{2} \mathrm{CH}_{2} \mathrm{I}$ (5.0 eq, $1.1 \mathrm{mg}, 4.5 \mu \mathrm{mol}$ ) was performed, product 11 was obtained in $79 \%$ yield $(0.95 \mathrm{mg}, 0.71 \mu \mathrm{mol}) .{ }^{1} \mathrm{H} \mathrm{NMR}\left(\mathrm{CS}_{2}, 400\right.$ $\mathrm{MHz}) \delta 0.60\left(\mathrm{t}, J=7.2 \mathrm{~Hz}, 3 \mathrm{H}, \mathrm{CH}_{3} \mathrm{CH}_{2} \mathrm{OCO}\right), 0.64(\mathrm{t}, J=7.0 \mathrm{~Hz}, 3 \mathrm{H}$, $\left.\mathrm{CH}_{3} \mathrm{CH}_{2} \mathrm{OCO}\right), 1.55-1.76\left(\mathrm{~m}, 4 \mathrm{H}, \mathrm{CH}_{2}\right), 1.79-2.17\left(\mathrm{~m}, 7 \mathrm{H}, \mathrm{CH}_{2}\right), 2.45(\mathrm{dt}, J=4.8,12.8$ $\mathrm{Hz}, 1 \mathrm{H}, \mathrm{CH} H$ ), 3.29 (s, 2H, $\mathrm{PhCH}_{2}$ ), 3.32 (d, $\left.J=13.0 \mathrm{~Hz}, 1 \mathrm{H}, \mathrm{PhCH} H\right), 3.44$ (q, $J=7.2 \mathrm{~Hz}$, $2 \mathrm{H}, \mathrm{CH}_{3} \mathrm{CH}_{2} \mathrm{OCO}$ ), 3.51 (q, $J=7.2 \mathrm{~Hz}, 2 \mathrm{H}, \mathrm{CH}_{3} \mathrm{CH}_{2} \mathrm{OCO}$ ), 3.55 (s, 5H, Cp), 3.59 (d, $J=13.0$ $\mathrm{Hz}, 1 \mathrm{H}, \mathrm{PhCH} H), 6.43-6.59$ (m, 8H, Ph), 6.70 (d, $J=7.0 \mathrm{~Hz}, 2 \mathrm{H}, \mathrm{Ph}), 6.76$ (t, $J=7.5 \mathrm{~Hz}, 1 \mathrm{H}$, $\mathrm{Ph}), 6.97$ (t, $J=7.8 \mathrm{~Hz}, 2 \mathrm{H}, \mathrm{Ph}), 7.44(\mathrm{~d}, J=7.4 \mathrm{~Hz}, 2 \mathrm{H}) ;{ }^{13} \mathrm{C} \mathrm{NMR}\left(\mathrm{CS}_{2}, 100 \mathrm{MHz}\right) \delta 15.10$ (1C), 15.13 (1C), 23.88 (1C), 24.24 (1C), 34.17 (1C), 34.34 (1C), 41.97 (1C), 42.61 (1C), 49.88 (1C), 50.85 (1C), 54.43 (1C), 54.51 (1C), 55.76 (1C), 55.77 (1C), 58.63 (1C), 60.44

(1C), 60.46 (1C), $69.82 \quad(5 \mathrm{C}), 91.18$ (1C), 91.60 (1C), 91.76 (1C), 9207 (1C), 93.02 (1C), 127.11 (1C), 127.30 (1C), 127.49 (1C), 128.28 (2C), 128.41 (2C), 128.46 (2C), 129.08 (2C), 130.43 (2C), 130.47 (2C), 136.60 (1C), 136.66 (1C), 142.55 (1C), 142.57

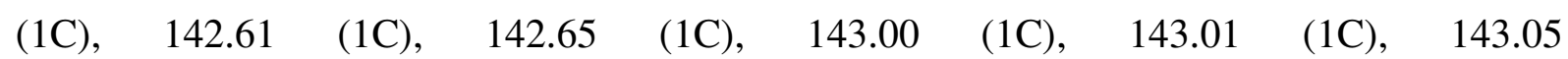
(1C), 143.09 (1C), 143.12 (1C), 143.13 (1C), 143.66 (1C), 143.69 (1C), 143.73 (1C), 144.33 (1C), 144.46 (1C), 144.61 (1C), 144.63 (1C), 144.72 (1C), 144.75 (1C), 144.84 (1C), 145.04 (1C), 146.78 (1C), 146.87 (1C), 146.91 (1C), 147.02 (1C), 147.06 (1C), 147.72 (1C), 147.77 (1C), 147.79 (1C), 147.80 (1C), 147.81 (1C), 147.87 (1C), 147.88 (1C+1C), 147.90 (1C), 147.92 (1C), 148.14 (1C), 148.16 (1C), 148.23 (1C), 148.28 (1C), 148.32 (1C), 152.17 (1C), 152.22 (1C), 152.28 (1C), 152.38 (1C), 152.43 (1C), 152.78 (1C), 152.98 (1C), 153.05 (1C), 153.29 (1C), 153.38 (1C), 171.60 (1C), 171.80 (1C); HRMS (APCI+) calcd for $\mathrm{C}_{97} \mathrm{H}_{46}{ }^{56} \mathrm{Fe}^{16} \mathrm{O}_{4}\left(\mathrm{M}^{+}\right):$1330.2746, found: 1330.2697 .

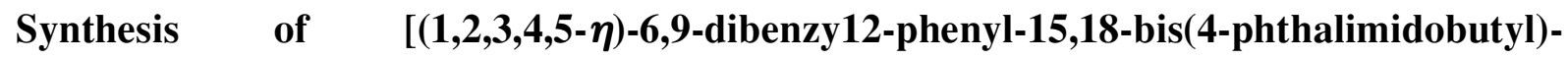
9,12,15,18-tetrahydro $\left(\mathrm{C}_{60}-I_{\mathrm{h}}\right)[5,6]$ fulleren-1 $(6 H)$-yl $]\left(\eta^{5}\right.$-cyclopentadienyl)iron(II) $<\mathrm{Fe}\left[\mathrm{C}_{60}\left(\mathrm{PhCH}_{2}\right)_{2} \mathrm{Ph}\left(\mathrm{C}_{6} \mathrm{H}_{4}(\mathrm{CO})_{2} \mathrm{NCH}_{2} \mathrm{CH}_{2} \mathrm{CH}_{2} \mathrm{CH}_{2}\right)_{2}\right]\left(\mathrm{C}_{5} \mathrm{H}_{5}\right)(12)>$.

The procedures $\left(\mathrm{RX}=\mathrm{C}_{6} \mathrm{H}_{4}(\mathrm{CO})_{2} \mathrm{NCH}_{2} \mathrm{CH}_{2} \mathrm{CH}_{2} \mathrm{CH}_{2} \mathrm{Br}\right)$ described above was performed to obtain 12. Yield: 70\%; ${ }^{1} \mathrm{H} \mathrm{NMR}\left(\mathrm{CDCl}_{3}, 400 \mathrm{MHz}\right) \delta 2.05-2.20(\mathrm{~m}, 8 \mathrm{H}$, butyl), 2.80-3.10 (m, 4H, butyl), 3.80-3.95 (m, 4H, butyl), 4.05-4.20 (m, 4H, $\left.\mathrm{CH}_{2} \mathrm{Ph}\right), 4.40$ (s, 5H, Cp), 7.20-7.40 (m, 7H, Ph), $7.52(\mathrm{~m}, 4 \mathrm{H}, \mathrm{Ph}), 7.70\left(\mathrm{~m}, 2 \mathrm{H}, \mathrm{C}_{6} \mathrm{H}_{4}\right), 7.73$ (m, 2H, Ph), $7.74(\mathrm{~m}$, $\left.2 \mathrm{H}, \mathrm{C}_{6} \mathrm{H}_{4}\right), 7.79\left(\mathrm{~m}, 2 \mathrm{H}, \mathrm{C}_{6} \mathrm{H}_{4}\right), 7.86\left(\mathrm{~m}, 2 \mathrm{H}, \mathrm{C}_{6} \mathrm{H}_{4}\right), 8.21(\mathrm{~d}, 2 \mathrm{H}, o-\mathrm{Ph}) ;{ }^{13} \mathrm{C} \mathrm{NMR}\left(\mathrm{CDCl}_{3}, 100\right.$ $\mathrm{MHz}) \delta 24.97$ (1C), 25.05 (1C), 29.08 (1C), 29.35 (1C), 37.51 (1C), 37.67 (1C), 42.17 (1C), 42.73 (1C), 49.65 (1C), 50.51 (1C), 54.51 (1C), 54.57 (1C), 55.60 (1C), 55.62 (1C), 58.57 (1C), 69.50 (5C), 91.32 (1C), 91.80 (1C), 92.00 (1C), 92.16 (1C), 92.89 (1C), 123.28 
(2C), 123.32 (2C), 126.97 (1C), 127.21 (1C), 127.55 (1C), 128.15 (2C), 128.18 (2C), 128.40 (2C), 128.95 (2C), 130.40 (2C), 130.45 (1C), 130.51 (2C), 132.04 (2C), 132.12 (2C), 133.96 (2C), 133.99 (2C), 136.90 (2C), 142.44 (1C), 142.46 (1C), 142.48 (2C), 142.80 (1C), 142.82 (1C), 142.84 (1C), 142.93 (1C), 143.00 (1C), 143.69 (1C), 143.77 (1C), 143.96 (1C), 144.53 (1C), 144.62 (1C), 144.70 (1C), 144.71 (1C), 144.78 (1C), 144.91 (1C), 144.96 (1C), 145.16 (1C), 146.87 (1C), 146.95 (1C), 146.97 (1C), 147.07 (1C), 147.13 (1C), 147.75

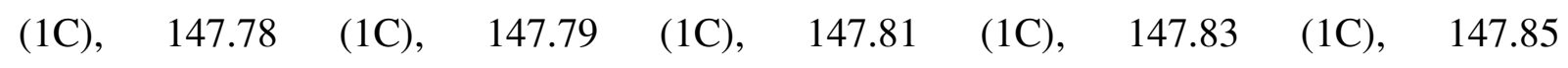
(1C), $147.92(1 \mathrm{C}+1 \mathrm{C}+1 \mathrm{C}), 148.01$ (1C), 148.10 (1C), 148.15 (1C), 148.23 (1C), 148.26 (1C), 148.30 (1C), 152.24 (1C), 152.31 (1C), 152.50 (1C), 152.57 (1C), 152.71 (1C), 152.94 (1C), 153.15 (1C), 153.24 (1C), 153.34 (1C), 153.43 (1C), 168.44 (2C), 168.50 (2C); HRMS (APCI+) calcd for $\mathrm{C}_{95} \mathrm{H}_{60}{ }^{56} \mathrm{Fe}^{16} \mathrm{O}_{10}\left(\mathrm{M}^{+}\right)$: 1504.2964, found: 1504.2922.

Synthesis of $\quad\left[(1,2,3,4,5-\eta)-6,9,12,15,18\right.$-pentabenzyl-9,12,15,18-tetrahydro $\left(\mathrm{C}_{60^{-}}\right.$ $\left.I_{\mathrm{h}}\right)[5,6]$ fulleren-1 $(6 H)$-yl $]\left(\eta^{5}\right.$-cyclopentadienyl)iron(II) $<\mathrm{Fe}\left[\mathrm{C}_{60}\left(\mathrm{PhCH}_{2}\right)_{5}\right]\left(\mathrm{C}_{5} \mathrm{H}_{5}\right)(13)>$.

A solution of tetrabutylammonium hydroxide (1.0 $\mathrm{M}$ in $\mathrm{MeOH}, 6.8$ equiv) was added to a solution of $\mathrm{Fe}\left(\mathrm{C}_{60} \mathrm{H}_{5}\right)\left(\mathrm{C}_{5} \mathrm{H}_{5}\right)\left(\mathbf{1}, 1.0\right.$ equiv) at room temperature $\left(21 \sim 26{ }^{\circ} \mathrm{C}\right)$. A color of the resulting solution changed from orange to dark red at a moment. After 5 min, the mixture was treated with benzyl bromide (286 equiv), resulting change of color from dark red to brown. HPLC analysis indicated the full consumption of the starting material within 10 minutes. Then the mixture was diluted with $8 \mathrm{~mL}$ of toluene/2-propanol $=1 / 1$, filtered with a pad of alumina and subjected to preparative HPLC separation. A collected fraction was concentrated to dryness and dried under reduced pressure to give a penta-alkylated product $\mathrm{Fe}\left[\left(\mathrm{C}_{60}\left(\mathrm{PhCH}_{2}\right)_{5}\right]\left(\mathrm{C}_{5} \mathrm{H}_{5}\right)(\mathbf{1 3})\right.$ in $47 \%$ yield. ${ }^{1} \mathrm{H} \mathrm{NMR}\left(\mathrm{CDCl}_{3}, 400 \mathrm{MHz}\right) \delta 4.30\left(\mathrm{~s}, 10 \mathrm{H}, \mathrm{CH}_{2}\right)$, $5.16(\mathrm{~s}, 5 \mathrm{H}, \mathrm{Cp}), 7.30(\mathrm{t}, 5 \mathrm{H}, p-\mathrm{Ph}), 7.34(\mathrm{t}, 10 \mathrm{H}, m-\mathrm{Ph}), 7.30(\mathrm{~d}, 10 \mathrm{H}, o-\mathrm{Ph}) ;{ }^{13} \mathrm{C}$ NMR

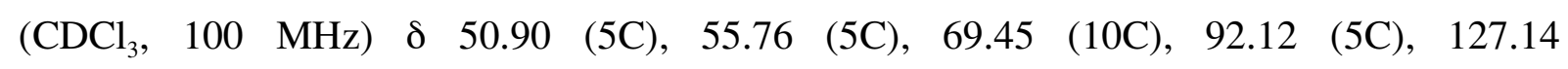
(5C), 128.32 (10C), 130.30 (10C), 136.67 (5C), 142.31 (10C), 144.55 (10C), 146.63 (5C), 147.46 (10C), 147.96 (5C), 152.28 (10C); HRMS (APCI+) calcd for $\mathrm{C}_{100} \mathrm{H}_{40}{ }^{56} \mathrm{Fe}$ $\left(\mathrm{M}^{+}\right)$: 1296.2479, found: 1296.2477 .

Synthesis of $\quad[(1,2,3,4,5-\eta)-6,9,12,15,18$-penta(3-etoxycarbonyl)propyl-9,12,15,18tetrahydro $\left(\mathrm{C}_{60}{ }^{-} I_{\mathrm{h}}\right)[5,6]$ fulleren-1 $\left.(6 H)-\mathrm{yl}\right]\left(\eta^{5}\right.$-cyclopentadienyl)iron(II) $<\mathrm{Fe}\left[\mathrm{C}_{60}\left(\mathrm{EtOOCCH}_{2} \mathrm{CH}_{2} \mathrm{CH}_{2}\right)_{5}\right]\left(\mathrm{C}_{5} \mathrm{H}_{5}\right)(14)>$.

The procedure described for 13 was performed to obtain 14. Complex 14 was prepared from 1 (1.5 mg, $0.0018 \mathrm{mmol}$ ) and $\mathrm{ICH}_{2} \mathrm{CH}_{2} \mathrm{CH}_{2} \mathrm{COOEt}(60 \mathrm{mg}, 0.246 \mathrm{mmol})$ in $\mathrm{PhCN}(2.0$ $\mathrm{mL})$. Yield: $1.5 \mathrm{mg}(1.0 \mathrm{mmol}, 58 \%)$. ${ }^{1} \mathrm{H} \mathrm{NMR}\left(\mathrm{CDCl}_{3}, 400 \mathrm{MHz}\right) \delta 1.29\left(\mathrm{t}, 10 \mathrm{H}, \mathrm{OCH}_{2} \mathrm{CH}_{3}\right)$, $2.32\left(\mathrm{~m}, 10 \mathrm{H}, \mathrm{C}_{60} \mathrm{CH}_{2} \mathrm{CH}_{2} \mathrm{CH}_{2}\right), 2.59\left(\mathrm{t}, 10 \mathrm{H}, \mathrm{C}_{60} \mathrm{CH}_{2} \mathrm{CH}_{2} \mathrm{CH}_{2}\right), 2.74\left(\mathrm{~m}, 10 \mathrm{H}, \mathrm{C}_{60} \mathrm{CH}_{2} \mathrm{CH}_{2} \mathrm{CH}_{2}\right.$ ), 4.19 (q, 10H, $\left.\mathrm{OCH}_{2} \mathrm{CH}_{3}\right), 4.77$ (s, 5H, Cp). ${ }^{13} \mathrm{C} \mathrm{NMR}\left(\mathrm{CDCl}_{3}, 100 \mathrm{MHz}\right) \delta 14.47\left(\mathrm{OCH}_{2} \mathrm{CH}_{3}\right)$, $23.72\left(\mathrm{C}_{60} \mathrm{CH}_{2} \mathrm{CH}_{2} \mathrm{CH}_{2}\right), 34.67\left(\mathrm{C}_{60} \mathrm{CH}_{2} \mathrm{CH}_{2} \mathrm{CH}_{2}\right), 42.68\left(\mathrm{C}_{60} \mathrm{CH}_{2} \mathrm{CH}_{2} \mathrm{CH}_{2}\right), 54.46\left(\mathrm{C}_{60}\left(\mathrm{sp}^{3}\right)\right)$, 
$60.60\left(\mathrm{OCH}_{2} \mathrm{CH}_{3}\right), 69.54(\mathrm{Cp}), 91.86(\mathrm{FCp}), 143.02\left(\mathrm{C}_{60}\right), 144.84\left(\mathrm{C}_{60}\right), 147.22\left(\mathrm{C}_{60}\right), 148.10$ $\left(\mathrm{C}_{60}\right), 148.41\left(\mathrm{C}_{60}\right), 153.28\left(\mathrm{C}_{60}\right), 173.24$ (COO); HRMS (APCI+) calcd for $\mathrm{C}_{95} \mathrm{H}_{60}{ }^{56} \mathrm{Fe}^{16} \mathrm{O}_{10}$ $\left(\mathrm{M}^{+}\right)$: 1416.3539, found: 1416.3551 .

Synthesis of $\left[(1,2,3,4,5-\eta)-6,9,12,15\right.$-tetrabenzyl-18-phenyl-9,12,15,18-tetrahydro $\left(C_{60^{-}}\right.$ $\left.I_{\mathrm{h}}\right)[5,6]$ fulleren-1 $(6 \mathrm{H})$-yl](tricarbonyl)rhenium $(\mathrm{I})<\mathrm{Re}\left[\mathrm{C}_{60}\left(\mathrm{PhCH}_{2}\right)_{4} \mathrm{Ph}\right](\mathrm{CO})_{3}(15)>$.

To a solution of $\mathrm{Re}\left[\mathrm{C}_{60}\left(\mathrm{PhCH}_{2}\right)_{2} \mathrm{PhH}_{2}\right](\mathrm{CO})_{3}(9.6 \mathrm{mg}, 7.7 \mu \mathrm{mol}, 1.0$ equiv) in $\mathrm{PhCN}$ $(2.0 \mathrm{~mL})$ was added a solution of tetrabutylammonium hydroxide in methanol $(1.0 \mathrm{M}, 18.4 \mu \mathrm{l}$, 2.4 equiv) at $22{ }^{\circ} \mathrm{C}$. A color of the solution changed from orange to dark red at a moment, indicating generation of a dianion species. After $10 \mathrm{~min}$, the mixture was treated with $\mathrm{PhCH}_{2} \mathrm{Br}(25.0 \mu \mathrm{l}, 27$ equiv) and the color of the reaction mixture immediately changed from dark red to brown. HPLC analysis indicated the full consumption of the starting material after 8 minutes. Then the mixture was diluted with $8 \mathrm{~mL}$ of toluene/2-propanol $=7 / 3$, filtered with a pad of alumina and subjected to preparative HPLC separation. A collected fraction was concentrated to dryness and dried under reduced pressure to give $\mathbf{1 5}$ as an orange solid (5.3 mg, 48\% yield). IR (powder) 2968 (w), 2933 (w), 2024 (s), 1940 (m), 1927 (m), 1494 (w), 1456 (w), 749 (m), 697 (s), 686 (m); ${ }^{1} \mathrm{H}$ NMR $\left(\mathrm{CDCl}_{3}, 400 \mathrm{MHz}\right) \delta 3.79$ (d, J=12.8 Hz, 2H, $\mathrm{PhCH} H$ ), 3.82 (d, $J=13.6 \mathrm{~Hz}, 2 \mathrm{H}, \mathrm{PhCH} H), 3.84(\mathrm{~d}, J=12.8 \mathrm{~Hz}, 2 \mathrm{H}, \mathrm{PhCH} H), 4.14$ (d, $J=13.6 \mathrm{~Hz}, 2 \mathrm{H}, \mathrm{PhCH} H), 7.21-7.74(\mathrm{~m}, 23 \mathrm{H}, \mathrm{Ph}), 8.19$ (d, $J=7.6 \mathrm{~Hz}, 2 \mathrm{H}, \mathrm{Ph}) ;{ }^{13} \mathrm{C} \mathrm{NMR}$ $\left(\mathrm{CDCl}_{3}, 100 \mathrm{MHz}\right) \delta 51.91$ (2C), 52.43 (2C), 55.87 (2C), 55.95 (2C), 58.74 (1C), 109.90 (2C), 110.75 (2C), 112.97 (1C), 127.26 (2C), 127.33 (2C), 127.51 (2C), 128.17 (4C), 128.22 (4C), 128.85 (2C), 129.32 (2C), 130.37 (4C), 130.40 (4C), 131.90 (2C), 132.49 (1C), 135.94 (2C), 135.99 (2C), 142.75 (2C), $142.81(2 \mathrm{C}+2 \mathrm{C}), 143.21$ (2C), 143.32 (2C), 143.40 (1C), 144.02 (2C), 144.05 (2C), 144.17 (2C), 144.50 (2C), 146.47 $\begin{array}{lllllll}(2 \mathrm{C}), & 146.49 & (1 \mathrm{C}), & 146.62 & (2 \mathrm{C}), & 147.43 & (2 \mathrm{C}),\end{array}$ $(2 \mathrm{C}+2 \mathrm{C}), 147.49(2 \mathrm{C}), 147.52$ (2C), 148.26 (2C), 148.36 (2C), $149.71(2 \mathrm{C}), 149.94$ (2C), 150.15 (2C), 150.39 (2C), 150.43 (2C), 192.33 (3C); HRMS (APCI+) calcd for $\mathrm{C}_{97} \mathrm{H}_{33}{ }^{187} \mathrm{ReO}_{3}\left(\mathrm{M}^{+}\right)$: 1432.1987 , found: 1432.1922 .

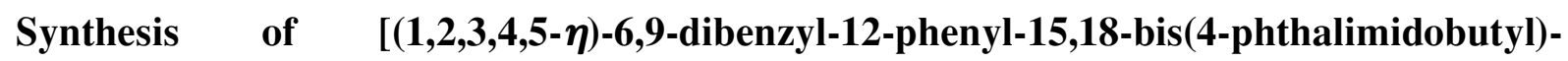
9,12,15,18-tetrahydro $\left(\mathrm{C}_{60}-I_{\mathrm{h}}\right)[5,6]$ fulleren-1 $(6 H)$-yl $]($ tricarbonyl $)$ rhenium(I) $<\mathrm{Re}\left[\mathrm{C}_{60}\left(\mathrm{PhCH}_{2}\right)_{2} \mathrm{Ph}\left(\mathrm{C}_{6} \mathrm{H}_{4}(\mathrm{CO})_{2} \mathrm{NCH}_{2} \mathrm{CH}_{2} \mathrm{CH}_{2} \mathrm{CH}_{2}\right)_{2}\right](\mathrm{CO})_{3}(16)>$.

To a solution of $\mathrm{Re}\left[\mathrm{C}_{60}\left(\mathrm{PhCH}_{2}\right)_{2} \mathrm{PhH}_{2}\right](\mathrm{CO})_{3}(9.6 \mathrm{mg}, 7.7 \mu \mathrm{mol}, 1.0$ equiv) in $\mathrm{PhCN}$ $(2.0 \mathrm{~mL})$ was added a solution of tetrabutylammonium hydroxide in methanol $(1.0 \mathrm{M}, 18.4 \mu \mathrm{l}$, 2.4 equiv) at $22{ }^{\circ} \mathrm{C}$. A color of the solution changed from orange to dark red at a moment, indicating generation of a dianion species. After $10 \mathrm{~min}$, the mixture was added $\mathrm{N}$-(4bromobutyl)phthalimide (54.3 mg, $0.193 \mathrm{mmol}, 25$ equiv) and heated at $80{ }^{\circ} \mathrm{C}$ for $30 \mathrm{~min}$. Color of the reaction mixture immediately changed from dark red to brown. HPLC analysis 
indicated the full consumption of the starting material. Then the mixture was diluted with 8 $\mathrm{mL}$ of toluene/2-propanol $=7 / 3$, filtered with a pad of silica and subjected to preparative HPLC separation. A collected fraction was concentrated to dryness and dried under reduced pressure to give $\mathbf{1 6}$ as an orange solid (9.3 mg, 73\% yield). ${ }^{1} \mathrm{H} \mathrm{NMR}\left(\mathrm{CDCl}_{3}, 400 \mathrm{MHz}\right)$ ठ 1.95-2.10 (m, 8H, butyl), 2.45-2.70 (m, 4H, butyl), 3.76-3.83 (m, 4H, butyl), $3.81(\mathrm{~d}, 1 \mathrm{H}$, $\mathrm{CH}_{2} \mathrm{Ph}$ ), 3.83 (s, 2H, $\mathrm{CH}_{2} \mathrm{Ph}$ ), 4.09 (d, $1 \mathrm{H}, \mathrm{CH}_{2} \mathrm{Ph}$ ), 7.28-7.65 (m, 18H, Ph), 7.68-7.72 (m, 4H, $\left.\mathrm{C}_{6} \mathrm{H}_{4}\right), 7.79-7.84\left(\mathrm{~m}, 4 \mathrm{H}, \mathrm{C}_{6} \mathrm{H}_{4}\right), 7.99(\mathrm{~d}, 2 \mathrm{H}, o-\mathrm{Ph}) ;{ }^{13} \mathrm{C} \mathrm{NMR}\left(\mathrm{CDCl}_{3}, 100 \mathrm{MHz}\right) \delta 24.04(1 \mathrm{C})$, 25.53 (1C), 28.81 (1C), 29.06 (1C), 37.44 (1C), 37.58 (1C), 44.58 (1C), 44.81 (1C), 51.95 (1C), 52.02 (1C), 54.94 (1C), 55.27 (1C), 55.96 (1C), 56.10 (1C), 58.81 (1C), 109.44 (1C), 110.23 (1C), 110.44 (1C), 112.18 (1C), 112.93 (1C), 123.21 (4C), 127.33 (1C), 127.45 (1C), 127.63 (2C), 128.16 (2C), 128.25 (1C), 128.34 (2C), 129.41 (2C), 130.54

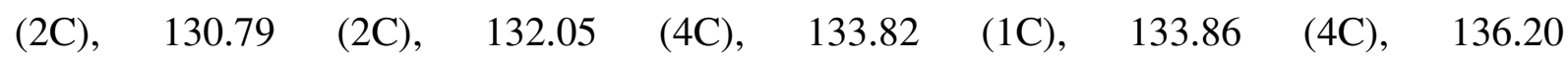
(1C), 136.22 (1C), 142.92 (1C), 142.95 (1C), 143.00 (1C), 143.07 (1C), 143.34

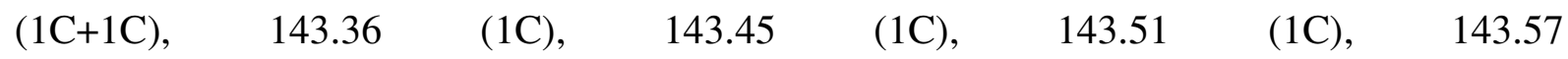
(1C), $144.29(1 \mathrm{C}+1 \mathrm{C}+1 \mathrm{C}), 144.32(1 \mathrm{C}+1 \mathrm{C}), 144.47(1 \mathrm{C}+1 \mathrm{C}+1 \mathrm{C}), 144.57(1 \mathrm{C}+1 \mathrm{C}), 146.75$ (1C), 146.82 (1C+1C), 146.90 (1C), 146.92 (1C), 147.68 (1C), $147.81(1 \mathrm{C}+1 \mathrm{C}), 147.86$ $(1 \mathrm{C}+1 \mathrm{C}), 148.50(1 \mathrm{C}+1 \mathrm{C}), 148.56(1 \mathrm{C}+1 \mathrm{C}), 148.61(1 \mathrm{C}), 150.22$ (1C), $150.28(1 \mathrm{C}), 150.35$ (1C), 150.47 (1C), 150.59 (1C), 150.92 (1C), 150.96 (1C+1C), 151.00 (1C), 151.01 (1C), 168.26 (2C), 168.31 (2C), 192.53 (3C); HRMS (APCI+) calcd for $\mathrm{C}_{107} \mathrm{H}_{44} \mathrm{~N}_{2} \mathrm{O}_{7}{ }^{187} \mathrm{Re}$ $\left(\mathrm{M}^{+}\right)$: 1653.2678, found: 1653.2644 . 
2. ${ }^{1}$ H NMR Spectra of 6 and Deprotonated Products

$\mathrm{Fe}\left[\mathrm{C}_{60}\left(\mathrm{PhCH}_{2}\right)_{2} \mathrm{PhH}_{2}\right]\left(\mathrm{C}_{5} \mathrm{H}_{5}\right)$ in $\mathrm{PhCN}-d_{5}$

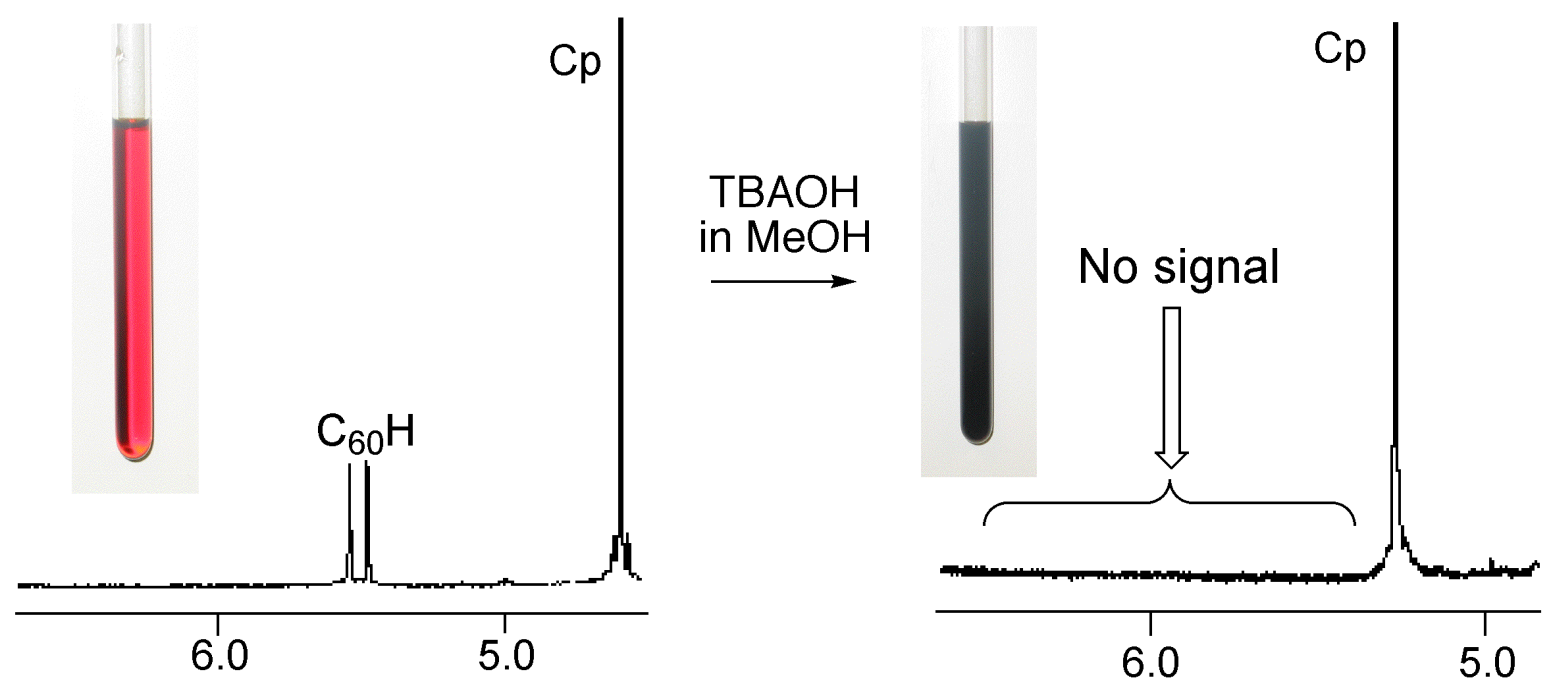

